\title{
Avaliação do estado nutricional em pacientes renais crônicos em hemodiálise
}

\author{
Assessment of nutritional status in chronic renal failure patients on hemodialysis \\ Taise Dobner ${ }^{1}$, Cristina Trevizan Telles ${ }^{2}$, Gabriela Pomatti ${ }^{2}$, Adriano Pasqualotti ${ }^{3}$, Luiz Antonio Bettinelli ${ }^{3}$ \\ ${ }^{1}$ Nutricionista graduada pelo Centro Universitário Franciscano. Mestre em Envelhecimento Humano pela Universidade de Passo Fundo (UPF). Residente do Programa de \\ Residência Multiprofissional do Hospital São Vicente de Paulo, em Saúde do Idoso. Passo Fundo, RS; ${ }^{2}$ Acadêmicas do curso de Enfermagem da UPF. Passo Fundo, RS; \\ ${ }^{3}$ Docentes do Programa de Pós-Graduação em Envelhecimento Humano da UPF. Passo Fundo, RS.
}

\section{RESUMO}

Objetivos: Avaliar o estado nutricional e fatores associados em pacientes renais crônicos em hemodiálise.

Métodos: Estudo do tipo transversal realizado no serviço de hemodiálise do Hospital São Vicente de Paulo, na cidade de Passo Fundo, Rio Grande do Sul. A avaliação antropométrica consistiu de peso (antes e após o período de hemodiálise), índice de massa corporal, circunferência muscular do braço, área muscular do braço, prega cutânea tricipital e espessura do músculo adutor do polegar (estas variáveis somente após o período de hemodiálise). Os exames bioquímicos realizados foram albumina, colesterol e transferrina. O recordatório alimentar foi aplicado para calorias totais e proteínas.

Resultados: Foram avaliados 90 pacientes, sendo 70 (77,8\%) homens. Pelo índice de massa corporal, 38 pacientes $(42,2 \%)$ tinham excesso de peso, $45(50 \%)$ eram eutróficos e sete $(7,8 \%)$ tinham baixo peso após a diálise. Excesso de peso foi estimado em $18(20 \%)$ dos pacientes pela prega cutânea tricipital e em nenhum paciente pelas outras medidas antropométricas utilizadas. Depleção nutricional foi identificada em 53 pacientes $(58,9 \%)$ pela prega cutânea tricipital, em $37(41,1 \%)$ pela circunferência muscular do braço, em $72(80 \%)$ pela área muscular do braço e em $84(95,4 \%)$ pela dosagem de albumina. O consumo alimentar de energia e o consumo de proteína estiveram abaixo dos padrões de referência e foram significativamente menores nos pacientes com excesso de peso.

Conclusões: Houve elevada prevalência de excesso de peso avaliado pelo índice de massa corporal, enquanto a maioria dos pacientes apresentou depleção nutricional nas demais variáveis antropométricas, com concomitante diminuição da albumina e da massa magra, após o período de hemodiálise.

DESCRITORES: DIÁLISE RENAL; ESTADO NUTRICIONAL; DESNUTRIÇÃO; FALÊNCIA RENAL CRÔNICA.

\section{ABSTRACT}

Aims: To assess the nutritional status and associated factors in chronic renal failure patients on hemodialysis.

Methods: A cross-sectional study was conducted in a referral nephrology center at Hospital São Vicente de Paulo, in the city of Passo Fundo, Rio Grande do Sul state. Anthropometric evaluation consisted of weight (before and after the period of hemodialysis), body mass index, arm muscle circumference, arm muscle area, triceps skinfold thickness and thickness of the thumb muscle (these only after the period of hemodialysis). Biochemical tests included albumin, transferrin and cholesterol. The dietary recall was applied to total calories and protein.

Results: Ninety patients were evaluated, of whom 70 (77.8\%) men. Using body mass index, 38 patients (42.2\%) were overweight, 45 (50\%) were normal and $7(7.8 \%)$ were underweight after dialysis. Overweight was estimated in $18(20 \%)$ patients by triceps skinfold and in no patient by the other anthropometric measures used. Nutritional depletion was identified in 53 patients (58.9\%) using the triceps skinfold, in $37(41.1 \%)$ by arm muscle circumference, in $72(80 \%)$ by arm muscle area and in $84(95.4 \%)$ by albumin dosage. Dietary intake of energy and protein intake were below the reference standards and were significantly lower in patients with overweight.

Conclusions: There was a high prevalence of overweight assessed by body mass index, whereas most patients had nutritional depletion in other anthropometric variables, with concomitant decrease in albumin and lean body mass, after the period of hemodialysis.

KEY WORDS: RENAL DIALYSIS; STATUS NUTRITIONAL; MALNUTRITION; CHRONIC KIDNEY FAILURE. 


\section{INTRODUÇÃO}

A mudança no perfil da mortalidade ocorrida nos últimos anos demonstra que as doenças crônicas não transmissíveis são responsáveis atualmente por cerca de $70 \%$ das mortes que ocorrem no Brasil. Entre estas, encontra-se a doença renal crônica (DRC). Dados epidemiológicos revelam que na América Latina a taxa de incidência em 2005 foi de 167,8 ppm (parte por milhão), tendo atingido 431 brasileiros em 2004. O último Censo da Sociedade Brasileira de Nefrologia revela que em 2011 o número de pacientes em diálise no país era de 91.314 pessoas. ${ }^{1-3}$

Tanto a DRC terminal (DRCT) quanto a terapia renal substitutiva acarretam alterações nutricionais importantes no paciente. A síndrome urêmica presente no portador de DRC está associada à perda de apetite e a efeitos gastrointestinais adversos, que resultam em redução do consumo alimentar, e à acidose metabólica associada à uremia, que promovem o catabolismo proteico. Para reverter esse quadro de acúmulo de substâncias tóxicas, uma das técnicas adotadas é a hemodiálise (HD), um processo intermitente que, entretanto, provoca acúmulo de outros produtos metabólicos e de substâncias inflamatórias, que pioram o estado nutricional. A inflamação é um achado importante nesses pacientes, e está relacionada à desnutrição e à ocorrência de doença arterial coronariana. ${ }^{4}$

Vários são os estudos que demonstram a prevalência de desnutrição nos pacientes em HD e os fatores que acarretam tal alteração, identificando o excesso de peso como fator de proteção contra a mortalidade. 5 Por outro lado, alguns pesquisadores iniciaram recentemente estudos sobre a prevalência de obesidade na área da DRCT, já que na população em geral o excesso de peso está relacionado à maior incidência de doenças crônicas não transmissíveis. ${ }^{6}$ Por isso, pesquisadores da área de nefrologia começam a investigar se a obesidade também não seria prejudicial aos pacientes em HD. ${ }^{7}$

Nessa perspectiva questiona-se: qual a prevalência de pacientes em HD com alteração do estado nutricional? E quais são os fatores associados a essas mudanças? Para responder a estes questionamentos, o objetivo deste estudo foi avaliar o estado nutricional e os fatores associados em pacientes em HD em um serviço de referência no norte do Rio Grande do Sul.

\section{MÉTODOS}

O estudo, do tipo transversal, foi realizado na cidade de Passo Fundo, no Serviço de Nefrologia do
Hospital São Vicente de Paulo, referência em HD. O projeto de pesquisa foi aprovado pelo Comitê de Ética e Pesquisa da Universidade de Passo Fundo sob número de protocolo 158.647. Foram incluídos no estudo os pacientes adultos acima de 18 anos com diagnóstico de DRCT em tratamento hemodialítico. Foram excluídos os pacientes que apresentavam problemas visuais ou neurológicos que impossibilitavam responder os questionários aplicados, os cadeirantes e os que não concordaram em assinar o termo de consentimento livre e esclarecido.

A avaliação antropométrica foi realizada após a sessão de HD, com exceção do peso, que foi aferido antes e após a sessão de HD. Utilizou-se uma balança Filizola com capacidade máxima de $180 \mathrm{~kg}$ e precisão de 100 g. O paciente foi pesado com o mínimo de roupa possível, descalço, em posição ereta e com os braços estendidos ao longo do corpo.

A altura foi obtida com estadiômetro acoplado na balança com o paciente ereto com os braços estendidos ao longo do corpo de costas para a parede em plano de Frankfurt. O Índice de Massa Corporal foi classificado segundo a Organização Mundial da $\mathrm{Saúde}^{8}$ e, para idosos, foi utilizada a classificação de Lipschitz. ${ }^{9}$ Optou-se por juntar as classificações de sobrepeso e obesidade em excesso de peso, e os diferentes graus de desnutrição em baixo peso, para fins estatísticos.

A circunferência do braço (CB) foi aferida no ponto médio entre o acrômio e o olécrano. Com o braço em $90^{\circ}$ ao lado do corpo localizou-se o ponto médio do braço do paciente, depois se estendeu o braço ao lado do corpo e a CB foi aferida com fita métrica inelástica com precisão de $1 \mathrm{~mm}$. Os cálculos de circunferência muscular do braço (CMB) e da área muscular do braço (AMB) foram realizados segundo Frisancho. ${ }^{10}$ Os resultados da CMB foram classificados em porcentagem de adequação, segundo os padrões de Blackburn e Thornton ${ }^{11}$ com base nos percentis de Frisancho. ${ }^{10}$

A prega cutânea triciptal (PCT) foi aferida no mesmo ponto médio da $\mathrm{CB}$, porém na face posterior do braço, utilizando plicômetro científico com precisão de $0,1 \mathrm{~mm}$ em posição longitudinal. A espessura do músculo adutor do polegar (EMAP) foi obtida segundo Lameu. ${ }^{12}$

Todas as medidas corporais foram feitas em triplicata e posteriormente foi feita uma média aritmética para sua classificação nos diferentes padrões de referência utilizados. As medidas da EMAP, CB e PCT foram realizadas no braço sem fístula arteriovenosa.

A coleta de exames bioquímicos foi realizada pela equipe do hospital antes do procedimento de HD. A transferrina foi considerada normal entre 200 e 
$300 \mathrm{mg} / \mathrm{dl}$ e alterada quando abaixo deste padrão. $\mathrm{O}$ colesterol sérico foi considerado normal quando menor que $200 \mathrm{mg} / \mathrm{dl}$ e alterado acima deste padrão. Para classificação da albumina denominou-se desnutrição grave quando a albumina foi $<2,0$, desnutrição moderada com valores entre 2,0 e 2,9, desnutrição leve entre 3 e 3,9 e normal quando $\geq 4 \mathrm{mg} / \mathrm{dl} .{ }^{13} \mathrm{O} \mathrm{Kt} / \mathrm{v}$ - onde (K) é a depuração de ureia do dialisador, multiplicada pelo tempo de tratamento ( $\mathrm{t}$ ) e dividida pelo volume de distribuição de ureia $(\mathrm{V})$ - foi considerado normal quando os valores eram $\geq 1,2$, padrão do serviço.

Para conhecer o consumo alimentar de calorias e proteína dos sujeitos da pesquisa utilizou-se como instrumento o recordatório alimentar de 24 horas e, para auxiliar na precisão das medidas consumidas, o registro fotográfico. ${ }^{14}$ Os dados coletados foram avaliados no Programa de Apoio a Nutrição - Nutwin, elaborado pela UNIFESP. ${ }^{15}$ Classificaram-se como normais a ingestão de $1,2 \mathrm{~g} / \mathrm{kg}$ de proteína, $35 \mathrm{kcal} / \mathrm{kg}$ para pacientes com menos de 60 anos e $30 \mathrm{kcal} / \mathrm{kg}$ para pacientes com mais de 60 anos. ${ }^{16}$ Dados complementares, como etiologia e convênio de realização da HD e etiologia da DRCT, foram obtidos dos prontuários dos pacientes.

Para a estruturação do banco de dados utilizaram-se os aplicativos Excel 2007 e Epi Info ${ }^{\mathrm{TM}} 3.5 .1$ e para as análises o programa estatístico R 2.10.0 para Windows. Para analisar os efeitos foram utilizados os testes de Mann-Whitney ou Kruskal-Wallis, qui-quadrado ou exato de Fisher e correlação ou Spearman. A avaliação da condição de normalidade foi realizada por meio dos testes de Kolmogorov-Smirnov e Shapiro-Wilk. O nível de significância utilizado foi de 0,05 .

\section{RESULTADOS}

Por ocasião da pesquisa, 132 pacientes eram atendidos no setor de HD do Hospital São Vicente de Paulo, dos quais 26 não aceitaram participar do estudo e 16 não atendiam aos outros critérios de inclusão. Dessa forma a amostra foi composta por 90 pacientes com diagnóstico de DRCT em HD de manutenção. Destes, 77,8\% $(\mathrm{n}=70)$ eram do sexo masculino. A média de idade foi de 53,52 \pm 16,34 anos, variando de 21 a 83 anos. Os pacientes idosos ( $>65$ anos) corresponderam a $42,2 \%$ da amostra. O tempo médio de HD foi de 42,99 $\pm 36,47$ meses, sendo o mínimo de um mês e o máximo de 180 meses.

Quanto à etiologia da doença renal, 36,7\% dos pacientes tinham a hipertensão arterial sistêmica como causa da DRCT. O principal convênio para realização da HD era o Sistema Único de Saúde (SUS) que atendia $86,7 \%$ dos pacientes (Tabela $\mathbf{1}$ ).
Tabela 1. Caracterização de 90 pacientes em hemodiálise de manutenção no Serviço de Nefrologia do Hospital São Vicente de Paulo, Passo Fundo, Rio Grande do Sul, no ano de 2013.

\begin{tabular}{llcc}
\hline Variáveis & \multicolumn{1}{c}{ Categorias } & n & $\%$ \\
\hline \multirow{2}{*}{ Sexo } & Feminino & 20 & 22,2 \\
& Masculino & 70 & 77,8 \\
\hline \multirow{2}{*}{ Etiologia } & Sistema Único de Saúde & 78 & 86,7 \\
& Outro & 12 & 13,3 \\
& Hipertensão arterial sistêmica & 33 & 36,7 \\
& Glomerulonefrite & 18 & 20,0 \\
& Diabetes mellitus & 15 & 16,7 \\
& Hipertensão arterial + Diabetes & 9 & 10,0 \\
& Outros & 9 & 10,0 \\
& Doenças autoimunes & 6 & 6,7 \\
\hline
\end{tabular}

$\mathrm{O}$ marcador $\mathrm{Kt} / \mathrm{V}$ estava alterado em 59 pacientes $(65,6 \%)$ no momento do estudo, tendo como média $1,32 \pm 0,28$. A média de peso pré HD foi de $72,87 \pm 13,97 \mathrm{~kg}$ e pós HD foi de $71,04 \pm 13,66 \mathrm{~kg}$, sendo, portanto, a média de ganho de peso interdialítico (GPID) $1,83 \pm 1,25 \mathrm{~kg}$.

O IMC revelou que metade da população estudada tinha alteração do estado nutricional, sendo 38 pacientes $(42,2 \%)$ com excesso de peso. Houve alteração das reservas musculares em um elevado número de pacientes, perceptíveis pela avaliação da CMB e da $\mathrm{AMB}$, atingindo $41,1 \%$ e $80 \%$, respectivamente (Tabela 2).

Tabela 2. Avaliação do estado nutricional de 90 pacientes em hemodiálise de manutenção no Serviço de Nefrologia do Hospital São Vicente de Paulo, Passo Fundo, Rio Grande do Sul, no ano de 2013.

\begin{tabular}{|c|c|c|c|}
\hline Variáveis & Categorias & $\mathbf{n}$ & $\%$ \\
\hline \multirow{3}{*}{$\begin{array}{l}\text { Índice de massa } \\
\text { corporal }\end{array}$} & Baixo peso & 7 & 7,8 \\
\hline & Eutrofia & 45 & 50,0 \\
\hline & Excesso de peso & 38 & 42,2 \\
\hline \multirow{3}{*}{$\begin{array}{l}\text { Prega cutânea } \\
\text { triciptal }\end{array}$} & Depleção & 53 & 58,9 \\
\hline & Eutrofia & 19 & 21,1 \\
\hline & Excesso de peso & 18 & 20,0 \\
\hline \multirow{2}{*}{$\begin{array}{l}\text { Circunferência } \\
\text { muscular do braço }\end{array}$} & Depleção & 37 & 41,1 \\
\hline & Eutrofia & 53 & 58,9 \\
\hline \multirow{2}{*}{$\begin{array}{l}\text { Área muscular do } \\
\text { braço }\end{array}$} & Depleção & 72 & 80,0 \\
\hline & Eutrofia & 18 & 20,0 \\
\hline \multirow{2}{*}{$\begin{array}{l}\text { Espessura do músculo } \\
\text { adutor do polegar }\end{array}$} & Normal & 78 & $86,7 \%$ \\
\hline & Diminuída & 11 & $12,3 \%$ \\
\hline \multirow[t]{4}{*}{ Albumina } & Depleção grave & 1 & 1,1 \\
\hline & Depleção moderada & 6 & 6,8 \\
\hline & Depleção leve & 77 & 87,5 \\
\hline & Normal & 4 & 4,5 \\
\hline
\end{tabular}


A média de IMC foi de $25,68 \pm 4,90 \mathrm{~kg} / \mathrm{m}^{2}$ e para a PCT a média foi $11,47 \pm 6,47 \mathrm{~mm}$. Considerando o percentual de adequação da $\mathrm{CMB}$ com relação ao percentil 50, a média de adequação do IMC foi de $92,77 \pm 13,94 \%$ e da AMB 74,03 $\pm 21,12 \%$. Na avaliação da EMAP encontrou-se que $78(86,7 \%)$ dos pacientes a apresentavam dentro dos padrões de referência, sendo a média $11,99 \pm 3,07 \mathrm{~mm}$.

Dois pacientes não tiveram a albumina avaliada, um o colesterol e outro a EMAP na rotina de exames. A dosagem de albumina apontou que 77 (87,5\%) sujeitos estudados tinham depleção leve. A transferrina esteve alterada em $74(82,2 \%)$ pacientes, com uma média de $163,64 \pm 40,42 \mathrm{mg} / \mathrm{dl}$. O colesterol apresentou-se alterado em $14(15,6 \%)$, sendo a dosagem média de $151,55 \pm 40,11 \mathrm{mg} / \mathrm{dl}$.

O recordatório alimentar de 24 horas revelou uma média de consumo de $1467 \pm 578 \mathrm{kcal}$ correspondendo a $21,4 \pm 9,6 \mathrm{kcal} / \mathrm{kg}$. O consumo de proteína foi de $0,92 \pm 0,52 \mathrm{~g} / \mathrm{kg}$, enquanto a taxa de aparecimento de nitrogênio proteico (PNA) revelou um consumo proteico um pouco mais elevado $(1,06 \pm 0,26 \mathrm{~g} / \mathrm{kg})$, entretanto também abaixo do padrão de referência.

Quando se avaliou a relação das diferentes classificações de IMC com as variáveis quantitativas, apresentadas na Tabela 3, notou-se que os pacientes com menor GPID foram os pacientes com baixo peso, com diferença significativa perante os outros grupos $(\mathrm{p}=0,016)$. Aqueles com excesso de peso apresentaram menor consumo de energia e proteínas por quilo de peso, com diferença estatística em relação aos demais grupos ( $p=0,010 \mathrm{e} p=0,035$, respectivamente). A PCT, a EMAP e a AMB foram diferentes estatisticamente entre as três classificações de IMC, com $p=0,001$ para as três variáveis. Todas essas medidas foram maiores no grupo com excesso de peso. A AMB e a EMAP foram menores no grupo com baixo peso e a PCT se apresentou menor para o grupo eutrófico pelo IMC. $\mathrm{O} \mathrm{Kt} / \mathrm{V}$ foi maior no grupo com baixo peso e com diferença significativa em relação aos demais grupos $(p=0,004)$. O grupo com excesso de peso apresentou o menor Kt/V.

\section{DISCUSSÃO}

Este estudo mostrou dados que vêm de encontro aos resultados do último Censo da $\mathrm{SBN},{ }^{3}$ o qual descreve que $84,9 \%$ dos pacientes realizam o tratamento dialítico pelo SUS, $57,3 \%$ são do sexo masculino e a principal etiologia é a hipertensão arterial sistêmica, sendo responsável por $35,1 \%$ dos casos. Segundo Schmidt et al., ${ }^{6}$ estima-se que $24 \%$ das mulheres e $17,3 \%$ dos homens com idade $\geq 20$ anos, e cerca de metade dos homens e mais da metade das mulheres

Tabela 3. Análise da relação entre o estado nutricional pelo índice de massa corporal e características antropométricas, laboratoriais e clínicas de 90 pacientes em hemodiálise no Serviço de Nefrologia do Hospital São Vicente de Paulo, Passo Fundo, Rio Grande do Sul, no ano de 2013.

\begin{tabular}{|c|c|c|c|c|c|c|c|}
\hline \multirow{3}{*}{ Variáveis } & \multicolumn{7}{|c|}{ Estado nutricional } \\
\hline & \multicolumn{2}{|c|}{ Baixo peso } & \multicolumn{2}{|c|}{ Eutrofia } & \multicolumn{2}{|c|}{ Excesso de peso } & \multirow{2}{*}{$\mathbf{p}$} \\
\hline & $\mathbf{n}$ & Posto & $\mathbf{n}$ & Posto & n & Posto & \\
\hline Idade & 7 & 58,9 & 45 & 41,5 & 38 & 47,8 & 0,202 \\
\hline Tempo de hemodiálise & 7 & 40,3 & 45 & 48,3 & 38 & 43,1 & 0,565 \\
\hline GPID & 7 & $18,9^{\mathrm{a}}$ & 45 & $49,5^{b}$ & 38 & $45,7^{\mathrm{b}}$ & 0,016 \\
\hline $\mathrm{kcal} / \mathrm{kg}$ & 7 & $50,8^{\mathrm{b}}$ & 45 & $52,9^{b}$ & 38 & $35,7^{\mathrm{a}}$ & 0,010 \\
\hline Proteína/Kg & 7 & $46,5^{b}$ & 45 & $52,2^{\mathrm{b}}$ & 38 & $37,3^{\mathrm{a}}$ & 0,035 \\
\hline PCT & 7 & $46,6^{a}$ & 45 & $35,7^{\mathrm{a}}$ & 38 & $56,9^{a}$ & 0,001 \\
\hline EMAP & 7 & $23,9^{a}$ & 44 & $38,4^{a}$ & 38 & $56,5^{a}$ & 0,001 \\
\hline CMB & 7 & $32,4^{\mathrm{b}}$ & 45 & $37,7^{\mathrm{b}}$ & 38 & $57,2^{a}$ & 0,001 \\
\hline AMB & 7 & $29,1^{\mathrm{a}}$ & 45 & $37,9^{\mathrm{a}}$ & 38 & $57,5^{a}$ & 0,001 \\
\hline $\mathrm{Kt} / \mathrm{v}$ & 7 & $76,0^{\mathrm{a}}$ & 45 & $45,1^{\mathrm{b}}$ & 38 & $40,3^{b}$ & 0,004 \\
\hline Transferrina & 7 & 37,9 & 45 & 45,1 & 38 & 47,4 & 0,668 \\
\hline Albumina & 7 & 45,9 & 45 & 42,6 & 38 & 46,6 & 0,767 \\
\hline PNA & 7 & 45,0 & 45 & 49,6 & 38 & 40,7 & 0,295 \\
\hline Colesterol sérico & 7 & 58,0 & 44 & 38,9 & 38 & 49,6 & 0,066 \\
\hline
\end{tabular}

Teste de Kruskal Wallis; Nível de significância para um $p \leq 0,05$.

Posto: Posição de referência utilizada pelo teste para comparação do nível de significância.

a, b: Letras diferentes indicam diferença significativa nas categorias analisadas do estado nutricional.

GPID: Ganho de peso interdialítico; PCT: Prega cutânea triciptal; EMAP: Espessura do músculo adutor do polegar; CMB: Circunferência muscular do braço; AMB: Área muscular do braço; PNA: Equivalente proteico de aparecimento de nitrogênio. 
com idade $\geq 60$ anos, relataram diagnóstico prévio de hipertensão. Associado a esse fato, há um elevado consumo de sódio e crescente excesso de peso nos últimos anos. A hipertensão arterial, se não tratada, pode levar em um curto espaço de tempo à DRCT, por lesão nas células renais. A prevalência de hipertensão aumenta progressivamente à medida que a função renal se deteriora, por isso, na fase dialítica, quase todos os nefropatas são hipertensos, contribuindo para o agravamento do risco de morte cardiovascular. ${ }^{17}$

Neste estudo $42,2 \%$ dos pacientes eram idosos, ao contrário de outros estudos que encontraram percentuais mais baixos, como na pesquisa de Calado et al. ${ }^{18}$ que descreve um percentual de idosos de $16,5 \%$. Dados coletados a partir de um sistema norteamericano revelaram que pacientes com mais de 75 anos de idade têm maiores taxas de incidência DRC do que os mais jovens, constituindo-se no segmento com mais rápido crescimento da população mundial com DRCT. ${ }^{19}$ Segundo Singh et al. ${ }^{20}$ os idosos exigem maiores cuidados e equipe mais especializada já que, além da DRCT, apresentam uma alta carga de condições crônicas de saúde, decréscimos na qualidade de vida e um alto risco de morte. As avaliações do presente estudo não revelaram diferenças dos marcadores em relação à idade.

Para verificar a adequação da HD, o serviço onde foram coletados os dados utiliza o $\mathrm{Kt} / \mathrm{V}$, dado importante já que, se a diálise for insuficiente, traz acúmulo de toxinas urêmicas, contribuindo para anorexia e desnutrição. ${ }^{21}$ No presente estudo, 65,6\% pacientes avaliados apresentaram alteração desse marcador. $\mathrm{O}$ grupo de pacientes com baixo peso apresentou o maior $\mathrm{Kt} / \mathrm{V}$, entretanto este marcador quando anormal associou-se significativamente com o grupo com excesso de peso. Este mesmo grupo apresentou a menor relação de consumo calórico e de proteínas por quilo de peso, o que pode estar associado ao Kt/V alterado. Barros et al., ${ }^{22}$ estudando 36 indivíduos em HD de manutenção, encontraram que os pacientes com excesso de peso, quando comparados àqueles com IMC $<25 \mathrm{~kg} / \mathrm{m}^{2}$, apresentavam níveis plasmáticos significativamente inferiores do peptídeo actil-grelina, um dos responsáveis por enviar ao sistema nervoso central estímulos para ingestão de alimentos.

Alguns trabalhos avaliaram o consumo alimentar de pacientes com DRC, encontrando-o abaixo das recomendações. Velludo et al. ${ }^{23}$ investigaram a alimentação de 39 pacientes em diálise de manutenção e encontraram que $67 \%$ tinham um consumo calórico inferior a $30 \mathrm{kcal} / \mathrm{kg} /$ dia. Favalessa et al. ${ }^{24}$ avaliaram 121 pacientes renais e observaram uma ingestão média de $21 \pm 7,8 \mathrm{kcal} / \mathrm{kg}$ e $0,9 \pm 0,3 \mathrm{~g} / \mathrm{kg}$ de proteína, ambos investigados pelo recordatório alimentar, assim como no presente estudo. Também como neste estudo, o PNA da investigação realizada por Favalessa et al. ${ }^{24}$ era maior que o do recordatório alimentar. Segundo Shinaberger et al. ${ }^{25}$ um consumo de proteína muito abaixo das necessidades $(<0,8 \mathrm{~g} / \mathrm{kg})$ foi associado à maior mortalidade, sendo que a melhor sobrevida foi associada ao consumo entre 1 e $1,4 \mathrm{~g} / \mathrm{kg}$, verificado através da cinética de ureia.

O GPID do presente estudo foi de $1,830 \mathrm{~kg}$. Segundo as recomendações da Kidney Disease Outcomes Quality Initiative (K/DOQI) da National Kidney Foundation, ${ }^{16}$ o GPID máximo deve ser de 5\% em relação ao peso seco; dessa forma, os pacientes do presente estudo apresentaram ganho de peso adequado entre as diálises. O controle no GPID está associado à adesão a dietoterapia, não significando ingerir menos calorias e sim restringir a ingestão de água e sódio. Nerbass et al., ${ }^{26}$ mostraram que o maior ganho de peso ocorreu em pacientes com algum grau de desnutrição aferidos pelo IMC e avaliação subjetiva. Já no presente estudo, os desnutridos pelo IMC foram os que menos ganharam peso entre as sessões de HD.

Vários são os estudos que investigaram o estado nutricional dos pacientes em $\mathrm{HD}$, tendo em vista que a alteração nutricional desses pacientes está ligada ao risco de complicações e mortalidade. $\mathrm{O}$ estudo de Castro et al. ${ }^{27}$ revelou que pelo IMC 39,3\% ( $\left.\mathrm{n}=99\right)$ tinham desnutrição ou risco nutricional, $28,6 \%(n=72)$ eram eutróficos e $32,1 \%(n=81)$ apresentavam excesso de peso. Para Cabral et al..$^{28}$ o percentual de pacientes desnutridos foi inferior a $18,9 \%$, assim como o de pacientes excesso de peso, enquanto $62,2 \%$ apresentavam-se eutróficos. O presente estudo encontrou metade dos pacientes com alteração do estado nutricional pelo IMC, sendo que a maior parte estava com o peso elevado para a altura, demonstrando que também estão sofrendo a transição nutricional como a população em geral, segundo dados do Vigitel Brasil 2011. ${ }^{29}$

Quando se observa o estado nutricional através dos marcadores CMB, AMB e PCT, o número de pacientes classificados com desnutridos neste estudo aumenta consideravelmente, atingindo 41,1\% (CMB) $80 \%$ (AMB) e 58,9\% (PCT). Outros estudos encontraram um percentual maior de desnutrição por esses marcadores, como o de Calado et al., ${ }^{18} \mathrm{com} 63 \%$ de desnutridos pela CMB e $63 \%$ pela PCT. Noori et al..$^{30}$ estudaram 126 pacientes e encontraram relação positiva entre a CMB e o percentual de massa magra, conhecido através de Absortometria Radiológica de Dupla Energia (DEXA). Também evidenciaram que os pacientes com maior CMB tinham melhor saúde 
mental, melhor qualidade de vida e maior sobrevida. Esses achados são importantes, pois esses marcadores de estado nutricional contribuem para a facilidade e fidedignidade das avaliações nutricionais, nos serviços que não contam com métodos mais sensíveis, como bioimpedâncias e DEXA.

Alguns autores tem proposto a utilização da EMAP como um marcador da avaliação nutricional, pois é o único músculo do corpo que poderia ser avaliado diretamente e é dificilmente afetado pelo estado de hidratação, podendo ser útil na avaliação de massa magra. Entretanto, poucos são os estudos recentes que utilizaram esse marcador em pacientes em HD. Um deles, de Pereira et al., ${ }^{31}$ avaliou a relação entre a EMAP e a força de preensão manual, um marcador já conhecido nas rotinas dialíticas, em 73 pacientes. Encontraram que os dois marcadores têm uma associação positiva, podendo o EMAP ser de grande valia na rotina de HD. Oliveira et al. ${ }^{32}$ investigaram a EMAP de 143 pacientes em HD e encontraram uma relação positiva entre esse marcador e IMC, AMB, CMB e massa magra. $\mathrm{O}$ presente estudo identificou diferença significativa do EMAP entre as três classificações de IMC, sendo os valores mais baixos encontrados nos pacientes de baixo peso e os mais elevados nos que apresentaram excesso de peso. Quando avaliada de forma qualitativa, a classificação de normalidade esteve associada ao excesso de peso. Porém ainda não há um ponto de corte específico para essa população, havendo necessidade de estudos controlados.

A desnutrição é usualmente definida como resultante da baixa ingestão de nutrientes, no entanto, no paciente renal crônico outros fatores estão envolvidos. Nesses pacientes, as proteínas plasmáticas tendem a estar diminuídas, em parte por causa das dietas restritivas e da anorexia urêmica. Assim, o paciente não ingere energia e proteína suficientes para sintetizar tecido muscular. A acidose metabólica também inibe a síntese de albumina, causa balanço nitrogenado negativo e degradação proteica. Estudos têm mostrado que a acidose é o estímulo inicial para a ativação e a transcrição de genes que codificam enzimas que participam de uma via responsável pela proteólise muscular. A acidose metabólica ainda pode estar associada à redução do anabolismo proteico. $\mathrm{O}$ estado inflamatório que costuma estar presente nos pacientes em HD também é importante fator contribuinte para a desnutrição. Estudos mostraram que algumas citocinas pró-inflamatórias exercem funções no apetite e no metabolismo de nutrientes, contribuindo para a desnutrição e para a alteração da composição corporal. , $33,34^{2}$
Quanto à albumina, Mutsert et al. ${ }^{35}$ avaliaram 700 pacientes para verificar a associação entre esta e a mortalidade, encontrando que a diminuição de $1 \mathrm{~g} / \mathrm{dl}$ de albumina associou-se a $47 \%$ de aumento no risco de morte em pacientes em HD. Essas alterações estavam associadas ao processo inflamatório e não ao estado nutricional, sugerindo que a albumina não poderia ser usada com precisão para o diagnóstico de avaliação nutricional. A maioria dos pacientes do presente estudo estava com a albumina diminuída, indicando desnutrição.

Os dados apresentados demonstram que as prevalências de excesso de peso e de desnutrição podem variar conforme a ferramenta utilizada para avaliação. No presente estudo o IMC revelou um elevado percentual de pacientes com peso por altura superior ao das recomendações, enquanto os marcadores que avaliaram tecido muscular ou adiposo demonstraram que o número de pacientes com depleção nutricional correspondia à maioria dos sujeitos estudados. Dessa forma, observou-se de maneira concomitante que os pacientes em HD podem estar sofrendo o processo conhecido como transição nutricional, identificado pelo elevado percentual de pacientes com excesso de peso e diminuição da massa magra, que pode estar ligada ao processo inflamatório e à inatividade física.

Essas duas variáveis não foram avaliadas no presente estudo, bem como a composição corporal através de métodos mais precisos, como a bioimpedância, que poderiam contribuir para a maior compreensão dos fatores associados às alterações nutricionais desses pacientes, indicando, portanto, limitações do estudo. Porém, mesmo sem essas avaliações, pode-se perceber a importância de uma avaliação nutricional periódica e do atendimento interdisciplinar nos serviços de nefrologia, para ajudar a prevenir e tratar as complicações decorrentes da diminuição de tecido muscular e/ou adiposo, como dietoterapia individualizada, treinamento muscular e aeróbico e monitoramento de problemas cardíacos, entre outros.

Conclui-se que houve elevada prevalência de excesso de peso, avaliado pelo IMC, assim como de depleção nutricional quando utilizados os outros marcadores, com concomitante diminuição da albumina e da massa magra nos pacientes após o período de HD. O presente estudo confirma ainda que a avaliação nutricional do paciente em HD deve ser constante e composta por diversos marcadores, para que em conjunto possam fornecer um diagnóstico completo dos portadores de DRCT que estão em tratamento hemodialítico. 


\section{REFERÊNCIAS}

1. Silva-Junior JB. As doenças transmissíveis no Brasil: tendências e novos desafios para o Sistema Único de Saúde. In: Ministério da Saúde, ed. Saúde Brasil 2008: 20 anos de Sistema Único de Saúde (SUS) no Brasil. Brasília: Ministério da Saúde; 2009.

2. Cusumano A, Gonzalez CB. Chronic kidney disease in Latin America: time to improve screening and detection. Clin J Am Soc Nephrol. 2008;3(2):594-600.

3. Sociedade Brasileira de Nefrologia (SBN) [Internet]. Censo de diálise 2011. [Acesso em 2012 mai 20]. Disponível em: http://www.sbn. org.br/pdf/censo_2011_publico.pdf

4. Leal VO, Leite Júnior M, Mafra D. Acidose metabólica na doença renal crônica: abordagem nutricional. Rev Nutr. 2008;21(1):93-103.

5. Vegine PM, Fernandes ACP, Torres MRSG, Silva MIB, Avesani CM. Avaliação de métodos para identificar desnutrição energético proteica de pacientes em hemodiálise. J Bras Nefrol. 2011;33(1):55-61.

6. Schmidt MI, Duncan BB, Silva GA, Menezes AM, Monteiro CA, Barreto SM, Chor D, Menezes PR. Health in Brazil 4. Chronic noncommunicable diseases in Brazil: burden and current challenges. The Lancet. 2011;377(9781)1949-61.

7. Honda H, Qureshi AR, Axelsson J, Heimburger O, Suliman M, Barany P, Stenvinkel P, Lindholm B. Obese sarcopenia in patients with end-stage renal disease is associated with inflammation and increased mortality. Am J Clin Nutr. 2007;86:633-8.

8. World Health Organization (WHO). Physical status: the use and interpretation of anthropometry. Report of a WHO Expert Committee WHO Technical Report Series 854.Geneva: World Health Organization; 1995.

9. Lipschitz DA. Screening for nutritional status in the elderly. Primary Care. 1994;21(1):55-67.

10. Frisancho AR. New norms of upper limb fat and muscle areas for assessment of nutritional status. Am J Clin Nutr.1981;34(11)2540-5.

11. Blackburn GL, Thornton PA. Nutritional assessment of the hospitalized patients. Med Clin North Am. 1979;63(5):11103-15.

12. Lameu EB, Gerude MF, Correa RC, Lima KA. Adductor policis muscle: a new anthropometric parameter. Rev Hosp Clín Fac Med S Paulo. 2004;59(2):57-62.

13. Martins C. Padrões de referência para exames laboratoriais. In: Riella MC, Martins CM. Nutrição e o Rim. Rio de Janeiro: Guanabara Koognan; 2001.

14. Zabotto CB, Viana RPT, Gil MF. Registro fotográfico para inquéritos dietéticos: utensílios e porções. SP/UNICAMP,Goiânia/UFG: Editora Metha; 1996.

15. Programa de Apoio à Nutrição. Versão 1.5. São Paulo: Departamento de Informática em Saúde, Universidade Federal de São Paulo; 2002.

16. National Kidney Foundation (NKF). K/DOQI Clinical practice guidelines for nutrition in chronic renal failure. K/DOQI, National Kidney Foundation. Am J Kidney Dis. 2000;35(2):1-140.

17. Bortolotto LA. Hipertensão arterial e insuficiência renal crônica. Rev Bras Hiper. 2008;15(3):152-5.

18. Calado IL, Silva AAM, França AKTC, Santos A, Salgado Filho N. Diagnóstico nutricional de pacientes em hemodiálise na cidade de São Luis (MA). Rev Nutr. 2009;22(5):687-96.

19. Collins AJ, Kasiske B, Herzog C, Chavers B, Foley R, Gilbertson D et al. Excerpts from the United States Renal Data System 2006 Annual Data Report. Am J Kidney Dis. 2007;49(1)1-296.

20. Singh P, Germain MJ, Cohen L, Unruh M. The elderly patient on dialysis: geriatric considerations. Nephrol Dial Transplant, 2013. doi: 10.1093/ndt/gft246.

21. Bonanni A, Mannucci I, Verzola D, Sofia A, Saffioti S, Gianetta E, Garibotto G. Protein-energy wasting and mortality in chronic kidney disease. Int J Environ Res Publ Healthy. 2011;8:1631-54.

22. Barros AF, Moraes C, Pinto MBS, Lobo JC, Mafra D. Is there association between acyl-ghrelin and inflammation in hemodialysis patients? J Bras Nefrol. 2013;35(2):120-6.

23. Velludo CM, Kamimura MA, Moreira PFP, Avesani C, Ribeiro FS, Vasselai P, Manfredi S, Canziani MEF, Draibe AS, Cuppari L. Estimativa de ingestão proteica de pacientes em hemodiálise: comparação entre registro alimentar e equivalente proteico de aparecimento de nitrogênio (PNA). J Bras Nefrol. 2007;29(4):245-51.

24. Favalessa E, Neitzke L, Barbosa GC, Molina MCB, Saralori LB. Avaliação nutricional e consumo alimentar de pacientes com insuficiência renal crônica. Rev Bras Pesq Saúde. 2009;11(4):39-48.

25. Shinaberger CS, Kilpatrick RD, Regidor DL, McAllister CJ, Greenland S, Kopple JD, Kalantar-Zadeh K. Longitudinal associations between dietary protein intake and survival in hemodialysis patients. Am J Kidney Dis. 2006;48(1):37-49.

26. Nerbass FB, Morais J, Santos RG, Kruger TS, Koene TT, Luz Filho HA. Fatores relacionados ao ganho de peso interdialítico em pacientes em hemodiálise. J Bras Nefrol. 2011;33(3):300-5.

27. Castro MCM, Oliveira FC, Silveira ACB, Gonzaga KBC, Xagoraris M, Centeno JR, Souza JAC. Importância da avaliação bioquímica mensal na triagem de pacientes com desnutrição em hemodiálise. J Bras Nefrol. 2010;32(4):352-58.

28. Cabral PC, Diniz AS, Arruda IKG. Avaliação nutricional de pacientes em hemodiálise. Rev Nutr. 2005;18(1):29-40.

29. BRASIL. Ministério da Saúde, Secretaria de Vigilância em Saúde. Vigitel Brasil 2011. Vigilância de fatores de risco e proteção para doenças crônicas por inquérito telefônico. Brasília: Ministério da Saúde; 2012.

30. Noori N, Kopple JD, Kovesdy CP, Feroze U, Sim JJ, Murali SB, Luna A, Gomez M, Luna C, Bross R, Nissenson AR, Kalantar-Zadeh K. Mid-arm muscle circumference and quality of life and survival in maintenance hemodialysis patients. Clin J Am Soc Nephrol. 2010;5(12):2258-68. 
31. Pereira RA, Caetano AL, Cuppari, L, Kamimura A. Adductor pollicis muscle thickness as a predictor of muscle strength in hemodialysis patients. Kidney Res Clin Prac. 2012;31(2):41-2.

32. Oliveira CM, Kubrusly M, Mota RS, Choukroun G, Neto JB, da Silva CA. Adductor pollicis muscle thickness: a promising anthropometric parameter for patients with chronic renal failure. J Ren Nutr. 2012;22(3):307-16.

33. Chung S, Koh ES, Shin SJ, Park CW. Malnutrition in patients with chronic kidney disease. Open J Inter Med. 2012;2:89-99.

34. Slee A. Exploring metabolic dysfunction in chronic kidney disease. Nutr and Metab. 2012;9(36):1-16.

35. Mutsert R, Grootendorst DC, Indemans F, Boeschoten EW, Krediet R, Dekker F. Association between serum albumin and mortality in dialysis patients partly explained by inflammation, and not by malnutrition. J Renal Nutr. 2009;19(2):127-35. 